\section{Radio-frequency ring resonators for self-referencing fiber-optic intensity sensors}

\author{
C. Vázquez, MEMBER SPIE \\ J. Montalvo \\ P. C. Lallana \\ Universidad Carlos III de Madrid \\ Escuela Politécnica Superior \\ Dpto. Tecnología Electrónica \\ Avenida Universidad 30 \\ 28911 Leganés, Madrid, Spain \\ E-mail: cvazquez@ing.uc3m.es
}

Abstract. A theoretical and experimental study of radiofrequency ring resonators $(R R)$ for referencing and improving the sensitivity of fiber-optic intensity sensors (FOS) is reported. The separation between lead and transducer losses in the FOS is solved by converting the light intensity fluctuations to be measured into RR losses that produce high amplitude variations in the proximity of the $R R$ resonance frequencies. Two different self-referencing techniques are developed. Via the definition of the measurement parameter $R_{M}$, sensor linearity and sensitivity are analyzed. A calibration using an optical attenuator is reported to validate the model. (c) 2005 Society of Photo-Optical Instrumentation Engineers. [DOI: $10.1117 / 1.1883566]$

Subject terms: fiber-optic sensors; self-referencing; intensity-based sensors; ring resonators.

Paper L040629 received Sep. 9, 2004; revised manuscript received Dec. 27, 2004; accepted for publication Jan. 18, 2005; appeared online Jan. 25, 2005; published online Apr. 12, 2005.

\section{Introduction}

Fiber-optic intensity sensors (FOS) based on multimode ${ }^{1}$ (MM) and single mode ${ }^{2}$ (SM) fibers need a self-referencing method to minimize the influences of long-term aging of source characteristics, as well as short-term fluctuations in optical power loss in the leads to and from the transducer. Time division, wavelength normalization, ${ }^{1,3}$ and frequencybased self-referencing methods $\mathrm{s}^{4,5}$ based on MM fibers, and a Michelson topology with SM fibers ${ }^{6}$ have been reported.

In this letter, we propose a novel frequency-based approach using a ring resonator (RR), with an improved sensitivity. Its principle and properties are discussed and tested.

\section{Theoretical Analysis}

The new sensing scheme is a RR and a FOS (see Fig. 1). The RR operates under an incoherent regime, so $\tau \gg T c$, where $T c$ is the source coherence time and $\tau$ is the loop transit time. The RR relative output power, $P_{3} / P_{1}$, is given by:

0091-3286/2005/\$22.00 @ 2005 SPIE $g \sqrt{\frac{K^{2}+[(1-2 \cdot K) \cdot H]^{2}+2 \cdot K \cdot(1-2 \cdot K) \cdot H \cdot \cos (\omega \cdot \tau)}{1+(K \cdot H)^{2}-2 \cdot K \cdot H \cdot \cos (\omega \cdot \tau)}}$,

$H=10^{-\alpha L / 10} \cdot A \cdot g \cdot F(m)$,

where $g=(1-\gamma), m$ is the measurand, $F(m)$ is the FOS calibration curve, $\gamma$ and $K$ are the coupler excess loss and coupling coefficient $\omega$ is the modulating signal, pulsation $\alpha$ is the fiber attenuation coefficient in $\mathrm{dB} / \mathrm{km}, A$ is an attenuation, and $L$ is the loop length. The FOS modulates the RR loss, $H$, and the output power frequency $P_{3} / P_{1}$, see inset of Fig. 1 for $K \in(0-0.5)$; there is a constant maximum if $\cos (\omega \tau)=+1$, and a dependent on $H$ minimum if $\cos (\omega \tau)$ $=-1$. The frequency normalization method is based on the sinusoidal modulation of the optical power source at two frequencies $f_{1}$ and $f_{2}$, as seen in Figs. 1 and 2. In this method, the measurement parameter is $R_{M 1}$ :

$R_{M 1}=\frac{\left|\frac{P_{3}}{P_{1}}\right|(\omega, \tau)}{\left|\frac{P_{3}}{P_{1}}\right|_{\mid \cos (\omega \tau)=1}}=\frac{\left|P_{3}\right|(\omega, \tau)}{\left|P_{3}\right|_{\mid \cos (\omega \tau)=1}}$.

The two ports normalization method uses a single frequency $\left(f_{1}\right)$, a coupler inside the RR for measuring $P_{4}$, and two down leads under identical external conditions; and the measurement parameter is $R_{M 2}$ :

$R_{M 2}=\frac{\left|P_{3}\right|(\omega, \tau)}{\left|P_{4}\right|_{\mid \cos (\omega \tau)=-1}}$.

The normalized sensitivity of the whole system is:

$\frac{1}{R_{M i}}\left(\frac{\partial R_{M i}}{\partial m}\right)=\frac{\partial R_{M i}}{R_{M i} \partial H}\left(\frac{\partial H}{\partial m}\right)=S_{M i} k_{1} S_{F}$

being $S_{F}=\delta \mathrm{F} / \delta m$ the FOS sensitivity, $k_{1}$ is a constant and $i=1,2$ for the frequency and two ports normalization method, respectively. This system sensibility is enhanced by $S_{M i}$. If $f_{1}$ is the resonance frequency, $S_{M 1}$ is given by:

$\frac{-(1-K)^{2}}{(1+K \cdot H) \cdot[K-(1-2 \cdot K) \cdot H]}$

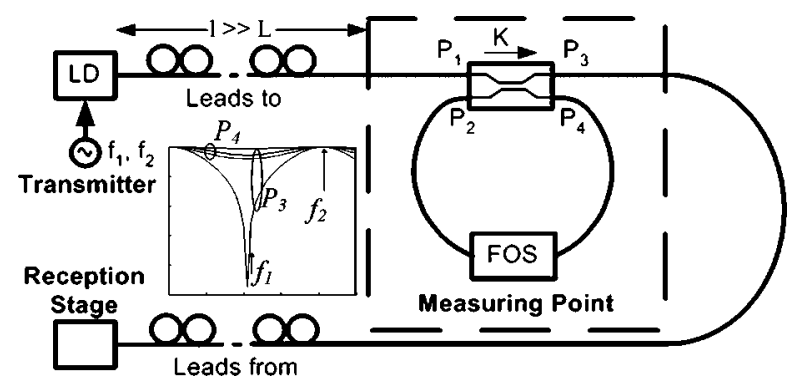

Fig. 1 General scheme of a RR for self-referencing FOS. Inset shows RR relative output powers versus frequency for different $H$ values to illustrate operation. 


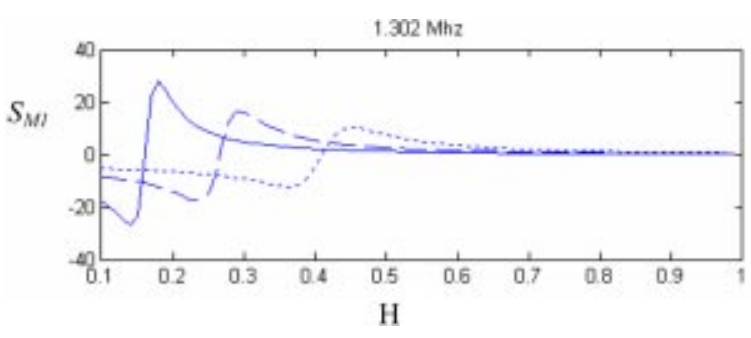

Fig. 2 Normalized sensitivity, $S_{M 1}$, versus $H$, in the frequency normalization method. $f_{1}=1,302 \mathrm{MHz}, L=1067 \mathrm{~m}, \gamma=0.05$, and $\left(\_\right)$: $K=0.11,(--): K=0.17,(\ldots): K=0.22$.

So $S_{M 1}$ tends to $\infty$ if $H \rightarrow H_{0}=K /(1-2 K)$,

The presence of noise limits the real value of the sensitivity. $S_{M 1}$ is plotted at Fig. 2, for a $f_{1}$ frequency of $1,302 \mathrm{MHz}$, in a RR with a loop length of $1067 \mathrm{~m}$. There is an inflection point for every $K$ at the $H_{0}$ value. For every quiescent point, a certain $K$ can be selected for achieving high sensitivities. $S_{M 2}$ behaves quite similar to $S_{M 1}$.

\section{Measurements}

The experimental setup is made of a LD of $1.5 \mu \mathrm{m}$, with 5 $\mathrm{MHz}$ linewidth, internally modulated with a signal coming from the tracking generator of a RF spectrum analyzer. The sensing scheme (see Fig. 1) is made of a polarization maintaining $2 \times 2$ variable ratio fiber coupler with pigtails of 1 $\mathrm{m}, 1067 \mathrm{~m}$ of standard SM fiber, and an attenuator simulating the FOS. $f_{1}$ is $1.302 \mathrm{MHz}, f_{2}$ is $1.207 \mathrm{MHz}$, and $K$ $=0.22$. The calibration curves, for both self-referencing methods, are reported in Fig. 3. There is a great agreement between theory and measurements, and the system reveals good sensitivity compared to other topologies; ${ }^{5}$ even though $f_{1}$ is not in the resonance frequency. Measurements variations, around $4 \%$, could be improved using a low coherence source in order to decrease the source induced noise.

\section{Conclusions}

Two different self-referencing methods for intensity fiberoptic sensors are described and their sensitivities are theo-

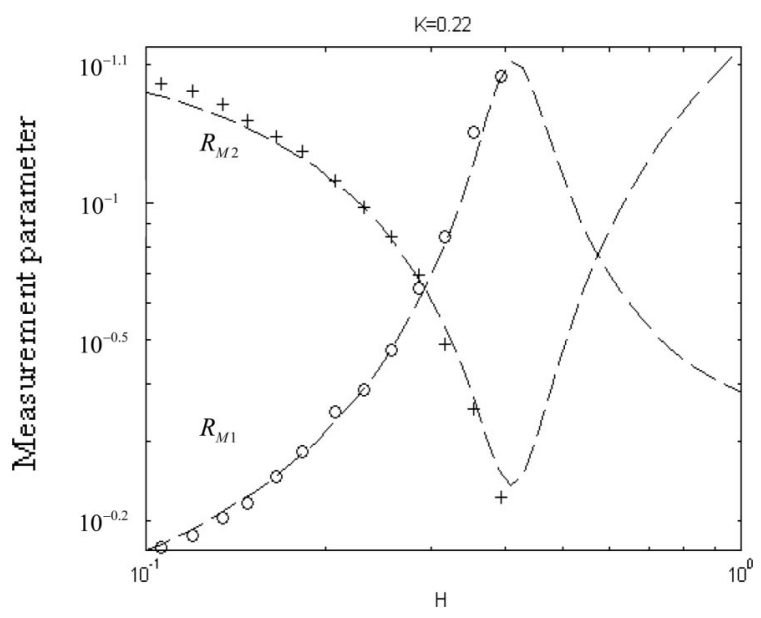

Fig. 3 Calibration curves for $K=0.22$ : measurements $R_{M 1}(\bigcirc)$ and $R_{M 2}(+)$ and simulations (dashed line).

retically analyzed. The proposed scheme, using RR operating under incoherent regime, is flexible because the operation point and sensitivity is controlled by a coupling coefficient. Experimental calibration curves are reported validating the utility of the model developed. This configuration has a better sensitivity to other topologies.

\section{Acknowledgments}

We wish to thank J. M. Sánchez-Pena and S. Vargas. This work was supported by Comision Interministerial de Ciencia y Tecnología (TIC2003-03783).

\section{References}

1. J. W. Berthold III, "Historical review of microbend fiber-optic sensors," J. Lightwave Technol. 13, 1193-1199 (1995).

2. T. Abe, Y. Mitsunaga, and H. Koga, "A strain sensor using twisted optical fibers," J. Lightwave Technol. 7, 525-529 (1989).

3. W. B. Spillman and J. R. Lord, "Self-referencing multiplexing technique for fiber-optic intensity sensors," J. Lightwave Technol. LT-5, 865-869 (1987)

4. P. Sixt, G. Kotrotsios, L. Falco, and O. Parriaux, "Passive fiber FabryPerot filter for intensity-modulated sensors referencing," J. Lightwave Technol. LT-4, 926-932 (1986).

5. J. M. Baptista, J. L. Santos, and A. S. Lage, "Mach-Zehnder and Michelson topologies for self-referencing fiber optic intensity sensors," Opt. Eng. 39, 1636-1644 (2000).

6. J. M. Baptista, S. Abad, G. M. Rego, L. A. Ferreira, F. M. Araujo, and J. L. Santos, "Wavelength multiplexing of frequency-based selfreferenced fiber optic intensity sensors," Opt. Eng. 43, 702-707 (2004). 